OPEN ACCESS

Edited by:

Lars Kaestner,

Saarland University, Germany

Reviewed by:

Angela Risso,

University of Udine, Italy

Teresa Tiffert,

University of Cambridge,

United Kingdom

*Correspondence:

Ryszard Grygorczyk

ryszard.grygorczyk@umontreal.ca

Specialty section:

This article was submitted to Membrane Physiology and Membrane

Biophysics,

a section of the journal

Frontiers in Physiology

Received: 27 September 2017 Accepted: 14 December 2017 Published: 22 December 2017

Citation:

Grygorczyk R and Orlov SN (2017)

Effects of Hypoxia on Erythrocyte

Membrane Properties -Implications

for Intravascular Hemolysis and

Purinergic Control of Blood Flow.

Front. Physiol. 8:1110.

doi: 10.3389/fphys.2017.01110

\section{Effects of Hypoxia on Erythrocyte Membrane Properties - Implications for Intravascular Hemolysis and Purinergic Control of Blood Flow}

\author{
Ryszard Grygorczyk ${ }^{1 *}$ and Sergei N. Orlov ${ }^{2}$ \\ ${ }^{1}$ Medicine, Université de Montréal, Montreal, QC, Canada, ${ }^{2}$ Biology, M. V. Lomonosov Moscow State University, Moscow, \\ Russia
}

Intravascular hemolysis occurs in hereditary, acquired, and iatrogenic hemolytic conditions but it could be also a normal physiological process contributing to intercellular signaling. New evidence suggests that intravascular hemolysis and the associated release of adenosine triphosphate (ATP) may be an important mechanism for in vivo local purinergic signaling and blood flow regulation during exercise and hypoxia. However, the mechanisms that modulate hypoxia-induced RBC membrane fragility remain unclear. Here, we provide an overview of the role of RBC ATP release in the regulation of vascular tone and prevailing assumptions on the putative release mechanisms. We show importance of intravascular hemolysis as a source of ATP for local purinergic regulation of blood flow and discuss processes that regulate membrane propensity to rupture under stress and hypoxia.

Keywords: red blood cell, red cell ATP release, intravascular hemolysis, purinergic signaling, red cell membrane fragility, hypoxia-induced ATP release

\section{INTRODUCTION}

During the last three decades it has become increasingly clear that in addition to passive uptake and release of oxygen and metabolically-derived gases, the red blood cells (RBC) also exhibit diverse oxygen-sensitive responses that autonomously regulate their own properties and functions. For example, changes in partial oxygen tension $\left(\mathrm{PO}_{2}\right)$ trigger a shift in glucose consumption from the pentose phosphate pathway (PPP) in oxygenated cells to glycolysis in deoxygenated cells (Messana et al., 1996). This shift is adaptive, since hemoglobin undergoes constant oxidation to methemoglobin in oxygenated cells, and its reduction back to hemoglobin would be facilitated by the enhanced production of NADPH in the PPP. Deoxygenation affects active $\mathrm{Ca}^{2+}$ transport and cytoplasmic $\mathrm{Ca}^{2+}$ buffering in human RBC (Tiffert et al., 1993). It was also shown that $\mathrm{PO}_{2}$ has an impact on the activity of RBC monovalent ion transporters (Bogdanova et al., 2009). In RBC from several fish species, low $\mathrm{PO}_{2}$ is required for $\beta$-adrenoceptor-mediated stimulation of $\mathrm{Na}^{+} / \mathrm{H}^{+}$ exchanger and elevation of hemoglobin affinity for $\mathrm{O}_{2}$ via cytoplasm alkalization (Nikinmaa, 2002). In human $\mathrm{RBC}$ with mutated hemoglobin $(\mathrm{HbS}) \mathrm{K}^{+}, \mathrm{Cl}^{-}$co-transport has an abnormal $\mathrm{PO}_{2}$-dependence that probably contributes to the pathogenesis of sickle cell anemia (Brugnara et al., 1996). Apart from the above-listed enzymatic and ion transport pathways, mammalian $\mathrm{RBC}$ also show hypoxia-induced responses involved in regulation of blood flow. These include two different, likely complementary mechanisms: rapid reduction of blood viscosity via increased RBC deformability and, delayed but sustained increase of vessel's diameter via release of adenosine 
triphosphate (ATP) and purinergic receptor stimulated production of $\mathrm{NO}$ and other vasorelaxants in vascular endothelial cells.

\section{Effects of Hypoxia on Blood Viscosity and RBC Deformability}

Blood viscosity is determined by RBC flow properties that include adhesion, aggregation and deformability, i.e., ability to change shape under a given stress without hemolysing. Erythrocyte deformability affects blood flow in large blood vessels, due to the increased frictional resistance between fluid layers under laminar flow conditions. It also affects the microcirculatory blood flow significantly where erythrocytes are forced to pass through blood vessels with diameters smaller than their size. Numerous pathologies are associated with a decrease of RBC deformability. For our review it is important to note that increased blood viscosity in sickle cell anemia is caused by decreased RBC deformability due to gel formation of deoxygenated mutated hemoglobin $\mathrm{HbS}$ and its interaction with the cell membrane proteins determining membrane elasticity (for review see Yedgar et al., 2002; Diez-Silva et al., 2010; Viallat and Abkarian, 2014).

Modulation of blood viscosity by $\mathrm{PO}_{2}$ may involve different processes depending on the duration and extent of oxygenation/deoxygenation. On the long time scale, these may include gradual changes of RBC membrane surface charges, mainly due to reduction in sialic acid content, and deformability that correlate with age (Huang et al., 2011) and markers of oxidative stress (Mehdi et al., 2012). On the other hand, the impact of prolonged hypoxia on RBC membrane properties remains poorly defined which contrasts with well documented, e.g., altered protein sialation in other cells types such as tumor cells. However, brief dips to a lower range of $\mathrm{PO}_{2}$ as they occur in microcirculation, were recently found to have acute and significant impact on blood viscosity. Wei et al. (2016) reported that microinjection of $\mathrm{O}_{2}$ scavengers resulted in vasoactive mediator-independent capillary hyperemia in mice cerebral microcirculation. In additional experiments, using microfluidic channels of small $(5 \mu \mathrm{m})$ or large $(20 \mu \mathrm{m})$ size they assessed effect of oxygenation on erythrocyte flow velocity and shear-induced deformability, respectively. These experiments revealed that $\mathrm{O}_{2}$ depletion increased the velocity of erythrocyte flowing through the microfluidic channel due to increased RBC membrane deformability. Viewed collectively, these data demonstrate that in addition to the increment of vessel diameter (see below), elevation of blood flow in microcirculatory beds under hypoxic conditions might be achieved via $\mathrm{PO}_{2}$-dependent regulation of erythrocyte deformability as a key determinant of blood viscosity (Wei et al., 2016).

\section{Effects of Hypoxia on RBC ATP Release and Purinergic Regulation of Vascular Tone} Besides release of hemoglobin-associated nitric oxide (NO), hypoxia affects vascular tone via release of ATP from RBCs that, in turn, leads to activation of P2Y receptors on endothelial cells, stimulation of NO production and NO-mediated vasodilation (Dietrich et al., 2000; Wang et al., 2005; Ellsworth and Sprague,
2012; for review, see Ellsworth et al., 2009, 2016; Jensen, 2009; Luneva et al., 2015). In vitro studies have shown that shear stress, mechanical deformation and hypoxia are major stimuli of RBC ATP release (Bergfeld and Forrester, 1992; Sprague et al., 1996; Forsyth et al., 2012; Mairbäurl et al., 2013). These observations were confirmed using microbore capillaries (Fischer et al., 2003) and microfluidic channels (Price et al., 2004; Forsyth et al., 2011) demonstrating that shear stress per se is sufficient to trigger ATP release from RBC (Wan et al., 2011). Importantly, elevated ATP levels have also been found in vivo in venous effluent from exercising forearm muscle (Forrester, 1972; Ellsworth et al., 1995) and further augmented by exercise performed in hypoxia (Dietrich et al., 2000; González-Alonso et al., 2002). It has been demonstrated that only when the vessels were perfused with RBCs did venous effluent ATP level increase and the vessels dilate in response to low extraluminal $\mathrm{PO}_{2}$ (Dietrich et al., 2000). Recent studies have shown that RBC-mediated ATP release is reduced in aging humans, which may contribute to impaired vasodilation and oxygen delivery to skeletal muscle during hypoxemia with advancing age (Kirby et al., 2012). Attenuated ATP production and release during deoxygenation was also found in banked RBCs, likely contributing to augmented microvascular adhesion of transfused RBCs in vivo. These alterations could be substantially corrected by restoring glycolysis-mediated ATP production (Kirby et al., 2014). To the best of our knowledge the comparative analysis of the action of hypoxia on ATP release under baseline conditions and in RBC subjected to shear stress has not been performed yet.

\section{Search for Transporters Involved in ATP Release Triggered by Hypoxia}

Since mature mammalian RBCs are devoid of intracellular organelles and unable to secrete ATP via endoplasmic reticulumdependent exocytosis, it might be assumed that ATP release from RBC in hypoxic conditions is mediated by ATP-conducting channels (Praetorius and Leipziger, 2009). Cystic fibrosis transmembrane conductance regulator (CFTR), Pannexin-1 (Panex1), voltage dependent anion channel (VDAC), and other poorly defined VDAC-like maxi anion channels have been implicated in conductive ATP release in RBCs and in other cell types (Sprague et al., 1998; Sridharan et al., 2010, 2012).

Early reports have suggested that CFTR and other members of the superfamily of ATP-binding cassette transport proteins serve as a conductive pathway for ATP release, or regulate an associated ATP channels in several cell types, including RBCs (Reisin et al., 1994; Sugita et al., 1998). Since CFTR activity is regulated by cAMP-dependent PKA, it has been also hypothesized that shear stress- and hypoxia-induced ATP release involves activation of the cAMP signaling pathway (Sprague et al., 2007). Consistent with this hypothesis, ATP release has also been reported in response to other stimuli that elevate cAMP, such as agonists of prostacyclin (Montalbetti et al., 2011; Sridharan et al., 2012), or $\beta$-adrenergic receptors (Olearczyk et al., 2001; for review see Ellsworth and Sprague, 2012). However, subsequent studies by several independent groups with 
the patch clamp, lipid bilayer, and luminometry techniques, have not revealed any detectable CFTR-mediated or CFTRregulated ATP release in several epithelial and non-epithelial cells (Grygorczyk et al., 1996; Li et al., 1996; Reddy et al., 1996; Grygorczyk and Hanrahan, 1997a,b; Watt et al., 1998; Hazama et al., 1999). In particular, it was also determined that CFTR protein is absent in the RBCs (Hoffman et al., 2004) and the role of cAMP signaling pathway in stimulating RBC ATP release was contradicted by recent studies (Sikora et al., 2014; Keller et al., 2017).

VDAC mediates ATP movement across the outer mitochondrial membrane (Rostovtseva and Colombini, 1997), and similar large conductance anion selective channels have occasionally been found in the plasma membrane of several cells (Báthori et al., 2000). However, these plasma membrane maxi anion channels and VDAC were shown to be unrelated proteins (Sabirov et al., 2006, 2017). VDAC pore selectivity favors the flow of adenine nucleotides (ATP, ADP) and anionic metabolites, over molecules of the same size and charge. The flow of small cations, including $\mathrm{Ca}^{2+}$, proceeds at significant rates even for closed channel state (Colombini, 2012). Thus, presence of such large-conductance poorly selective channels in cell plasma membrane would perturb significantly cell homeostasis. Similar concerns may apply to other putative ATP channels (see below).

Connexins and the related pannexins, particularly Panx1 currently appear to be the most extensively investigated family of proteins reported to function as ATP conduits in a broad range of cell types (Romanello et al., 2001; Dando and Roper, 2009; Ma et al., 2009; Ransford et al., 2009; D’hondt et al., 2010; Lazarowski et al., 2011), including RBCs (Sridharan et al., 2010; Qiu et al., 2011; Chu et al., 2016). Nevertheless, several basic Panxlchannel properties, including single channel conductance, selectivity and regulatory mechanisms still remain unclear (Chiu et al., 2014). Systematic electrophysiological studies revealed that Panx1 is a relatively low conductance anion channel (unitary conductance of 68 to $75 \mathrm{pS}$ ) with negligible permeability to large anions (aspartate, glutamate, gluconate), (Ma et al., 2012; Chiu et al., 2014). In particular, no measurable Panex1 permeability to ATP was detected in taste buds and several heterologous expression systems (HEK-293, CHO, and SK-NSH cells) (Romanov et al., 2012). Thus, direct patch-clamp experiments to determine its selectivity so far did not provide convincing support for the involvement of these channels in ATP release. It has been suggested however, that permeability characteristics could change depending on how the channel was activated (Chiu et al., 2014).

Piezo 1 is a mechanosensitive non-selective cation channel expressed on RBC membrane. Gain-of-function mutations in Piezo 1 were linked to dehydrated hereditary stomatocytosis (Zarychanski et al., 2012; Albuisson et al., 2013). It was recently shown that Piezo 1 regulates mechanosensitive ATP release in RBCs by controlling the shear-induced $\mathrm{Ca}^{2+}$ influx (Cinar et al., 2015). Based on pharmacological data it was proposed that the release may involve CFTR and/or Pannexin 1 channels, but in the light of concerns discussed above, alternative pathways that might be modulated by intracellular $\mathrm{Ca}^{2+}$-elevation should be also considered (see below).
Regardless of the molecular nature of ATP-conducting channels, the main conceptual difficulty with such a release mechanism is that ATP permeation requires pore of large dimensions (0.6-1.1 nm, Sabirov and Okada, 2005), resulting in poor selectivity and large conductance (hundreds of $\mathrm{pS}$ ) for small ions such as $\mathrm{K}^{+}, \mathrm{Na}^{+}, \mathrm{Cl}^{-}$, and $\mathrm{Ca}^{2+}$, as exemplified by the well characterized VDAC channel. VDAC selectivity for ATP, ADP is based on steric constrains and charge distribution allowing to discriminate between large anions but it does not prevent permeation of small cations. Opening of such large, non-selective pores in the plasma membrane will result in significant influx of $\mathrm{Na}^{+}$and $\mathrm{Ca}^{2+}$ down their electrochemical gradients into the cytoplasm, posing an overwhelming challenge to normal cell homeostasis and survival (Akopova et al., 2012). Furthermore, in terms of energy expenditure, conductive ATP release would be a costly mechanism of intercellular signaling that requires action of energy-consuming transport processes to re-establish normal cellular $\mathrm{Ca}^{2+}, \mathrm{Na}^{+}$, and $\mathrm{K}^{+}$gradients. To the best of our knowledge, currently there is no example of a channel that on one hand would allow high permeability for anions as large as ATP and, on the other would prevent significant fluxes of small cations. None of the currently known putative plasma membrane ATP channels seems to be compatible with the requirement of preserving cell homeostasis. Therefore, the concept of such release mechanisms should be treated with caution.

\section{ATP Release by Hemolysis}

Hemolysis is an important source of extracellular ATP, and intravascular hemolysis occurs in vivo as a consequence of hypoxia and mechanical trauma to RBCs (Shaskey and Green, 2000; Mao et al., 2011; Mairbäurl, 2013). Although it has been also considered to be a potential factor contributing to stimulated ATP release in most previous in vitro investigations, its actual involvement has often not been assessed systematically. By paired measurements of ATP and free hemoglobin in each and every sample of human RBC supernatants Sikora et al. found that basal and stimulated ATP release not only correlated tightly with extracellular hemoglobin, but matched the levels expected from cell lysis and independently determined cell ATP content (Sikora et al., 2014, 2015). Unexpectedly, this was seen with all stimuli tested (hypotonic shock, shear stress, hypoxia) strongly indicating that, for each stimulus, the only source of extracellular ATP was cell lysis (Sikora et al., 2014; Luneva et al., 2016). Surprisingly, stimulation of cAMP pathway had no effect on RBC ATP release, which remained at the basal level observed with unstimulated cells. The report triggered a significant debate in the field with opposing views presented by Kirby et al. (2015) and Sikora et al. (2015). Absence of cAMP-regulated ATP release was recently confirmed by Keller et al. who also showed using Panex1 $1^{-/}$mice model that Panexl has no role in exercise performance, challenging assumptions about Panex1 role in ATP-dependent blood perfusion to exercising skeletal muscle (Keller et al., 2017). In the study by Sikora et al. the primary role of hemolysis in hypotonic shock-induced ATP release was confirmed more directly by simultaneous luminescence ATP imaging and infrared imaging of substrate-attached RBCs 
(Sikora et al., 2014). With luciferin-luciferase (LL) present in the extracellular solution these experiments identified single ATPreleasing cells and revealed that only lysing cells contributed to the release. This was seen as a flash of ATP-dependent LL luminescence around the cell followed, after some delay, with cell ghosting due to $\mathrm{Hb}$ leakage, Figure 1A. Individual cells showed variable duration of ATP release as might be expected for different number and/or size of lytic pores in the membrane, Figure 1B. Interestingly, time-course of ATP release correlated with time delay of cell ghosting, i.e., cells showing slower ATP release also displayed longer delay before ghosting, consistent with slower $\mathrm{Hb}$ leakage. This is in agreement with 3-to 4 -fold difference of their diffusion coefficients in water, supporting the view that release of $\mathrm{Hb}$ and ATP proceeds through the common pathway. The study demonstrated that at least in the case of hypotonic shock and cAMP/forskolin stimulation hemolysis is likely the only mechanism of RBC ATP release, and therefore processes that control RBC susceptibility to lysis will also contribute to modulation of ATP release (Sikora et al., 2014). In the light of this finding there is an urgent need to understand mechanisms underlying the hemolysis (Thomas, 2014).

RBC fragility, or propensity to hemolyse under osmotic or mechanical stress, is determined by properties of the cell membrane. It is comprised of a phospholipid bilayer and an underlying two-dimensional cytoskeleton, a network of
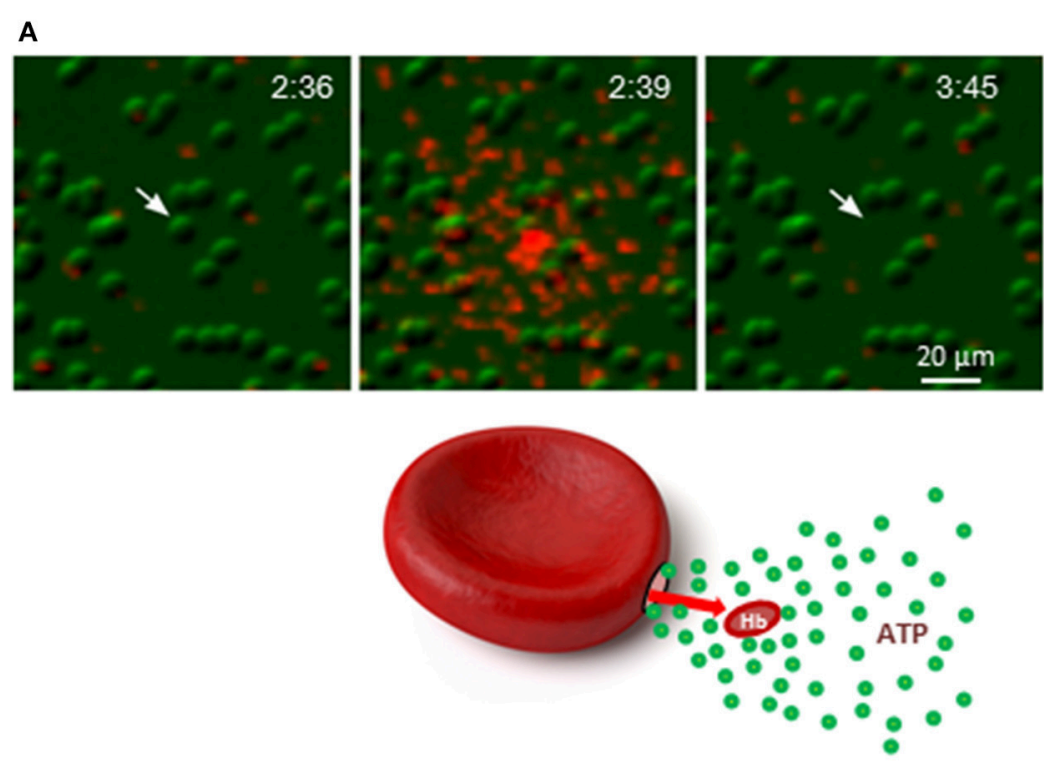

B

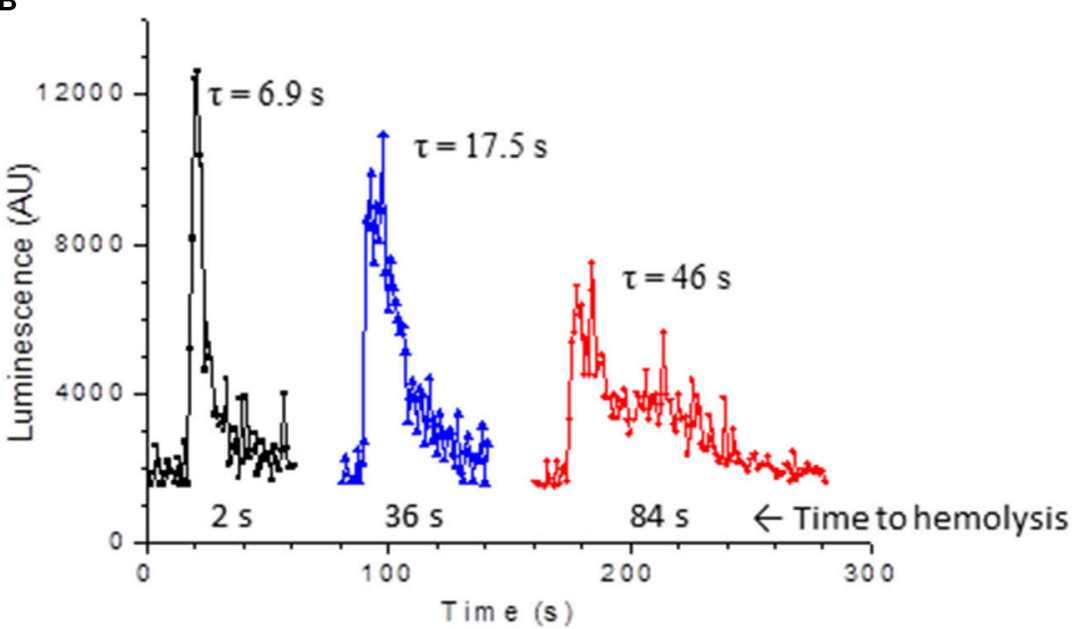

FIGURE 1 | ATP release due to lysis of single RBCs. (A), sequential infrared images of RBCs (green) that are overlaid with extracellular ATP-dependent bioluminescence from luciferin-luciferase reaction (red). Elapsed time is indicated in the right-upper corners (min:s). Twenty percent of hypotonic solution was introduced at time 0 and hypotonic shock-induced ATP release is shown on the center image. Ghosting of the RBC (indicated by arrow) due to Hb leakage occurred with a delay of about $66 \mathrm{~s}$. No ATP release from intact RBCs is evident. (B), three examples of ATP release time-course due to lysis of single RBCs such as shown in A. Duration of ATP release $\tau$ (in s) is indicated for each trace and corresponding delay from peak ATP release until cell ghosting is shown below. Adapted from Sikora et al. (2014). 
actin and $\alpha$ - and $\beta$-spectrin molecules that are held together by ankyrin. The membrane is stabilized by interactions of ankyrin with band 3, the major RBC membrane integral protein (Mohandas and Gallagher, 2008). The composite properties of the phospholipid bilayer and 2D cytoskeleton are responsible for the biconcave discocyte morphology of healthy RBC and membrane elastic and rheological properties. Disruptions of interactions between cytoskeletal components and/or integral membrane proteins change spectrin network density causing cell morphological changes and membrane fluctuations, affecting RBC deformability and fragility (Diez-Silva et al., 2010).

Under shear stress the RBC cell membrane deforms until the membrane reaches its "yield point." Beyond this threshold point, additional stress results in irreversible plastic deformation of the membrane, which accelerates with accumulation of microdefects in the membrane, leading to the cell destruction (Orbach et al., 2017). However stressed cells do not rupture immediately, the time-course of this process depends on the duration and extent of the stimuli. Li et al. (2013) showed that after exposure to brief pulse of cavitationinduced shear stress (i.e., during air bubble formation and collapse) RBC lysis is a 2-step process. It involves formation of nanopores followed by colloidal osmotic cell swelling until cell bursts. To reach that point, the nanopores in the membrane must remain for a sufficient time. In erythrocyte membrane the brush-like glycocalyx molecules by steric interactions may contribute to stabilization of nanopores. In their study pores of up to $1.6 \mu \mathrm{m}$ effective size were formed at rupture site that allowed diffusion of cellular content. Lysis occurred within few seconds to several tenths of seconds. Interestingly, in these experiments cells that ruptured showed irregular shape, indicating that changes in the $2 \mathrm{D}$ spectrin network and/or anchor points to plasma membrane are important factors enabling lytic pore formation ( $\mathrm{Li}$ et al., 2013).

\section{Role of RBC Aging and Membrane Vesiculation}

The circulating RBCs undergo a natural aging process occurring throughout their lifespan of about 120 days. The aged "senescent" cells are characterized by loss of cell surface area, cell morphology alterations, increased cell rigidity and aggregability, reduced level of cell membrane stomatin (band 7 protein), and translocation of phosphatidylserine (PS) to the cell surface. The surface membrane content of sialoglycoproteins and sialic acids which accounts for majority of the negative surface charge of RBC membrane is also reduced in aged $\mathrm{RBC}$ contributing to altered $\mathrm{RBC}$ membrane mechanical and electrical properties, receptormediated cellular interactions, immune responses and survival (Durocher et al., 1975; Huang et al., 2011). The aging of RBCs are also characterized by the formation and accumulation of microdefects in the RBC membrane which as mentioned above, makes them susceptible to stress. Indeed, Orbach et al. found that the cells that were destroyed under low mechanical stress were characterized by low deformability, high level of surface
PS, and reduced level of membrane stomatin, all properties consistent with aged senescent cells possessing augmented macrovesicle formation terminated by RBC lysis (Orbach et al., 2017).

The molecular mechanisms underlying the formation of the plasma membrane macrovesicles remain poorly understood. Changes in metabolic status and decrease of cellular ATP levels during prolonged deoxygenation induce RBC shape changes and increase membrane fluctuations. Membrane fluctuations are directly linked to binding of membrane bilayer to spectrin network which is actively controlled by ATP (Diez-Silva et al., 2010; Park et al., 2010). Low ATP-induced morphological changes are reversible upon restoration of normal cellular ATP levels. Thus, metabolic status of RBC might be important factor affecting $\mathrm{RBC}$ susceptibility to membrane vesiculation and RBC lysis. It was also well documented that RBC vesiculation is sharply potentiated by elevation of intracellular $\mathrm{Ca}^{2+}$ concentration $\left(\left[\mathrm{Ca}^{2+}\right]_{i}\right)$ via activation of scramblase and inhibition of flipase. These $\left[\mathrm{Ca}^{2+}\right]_{\mathrm{i}}$-dependent events results in a collapse of membrane phospholipid asymmetry and cytoskeleton detachment (for review see (Greenwalt, 2006; Alaarg et al., 2013). In early studies, Tiffert and co-workers observed that brief deoxygenation results in elevation of $\left[\mathrm{Ca}^{2+}\right]_{\mathrm{i}}$ up to $70 \%$ that was probably caused by $\mathrm{Ca}^{2+}$-ATPase inhibition (Tiffert et al., 1993).

Intravascular vesiculation process and associated hemolysis of senescent cells in healthy subjects was reassessed in recent study by Ciana et al. (2017). They showed that contrary to some earlier in vitro investigations vesiculation process of senescent RBCs removes membrane in a balanced way as a lipid bilayer vesicles containing membrane cytoskeleton. Moreover, the study suggests that in vivo vesiculation almost entirely occurs by active processing in the spleen producing progressively smaller but otherwise viable discoid shape cells. This agrees with the view that vesiculation is a self-protective mechanism to remove damaged membrane patches containing removal proteins, thereby postponing untimely elimination of healthy RBCs (Willekens et al., 2008). The study implies that in healthy subjects under normal conditions contribution of intravascular hemolysis to RBC clearance may be negligible. Therefore, intravascular hemolysis may occur only under particular conditions in the localized regions of the vasculature where elevated shear and hypoxia may arise, such as in the microvessels of skeletal muscle during intense exercise. It should be noted, however, that due to high ATP content of RBCs (1$5 \mathrm{mM}$ ) even negligibly small intravascular hemolysis may readily produce local ATP concentrations reaching $\sim 1 \mu \mathrm{M}$, sufficient for purinergic control of blood flow. For example, lysis of a single erythrocyte will result in ATP concentration of $1 \mu \mathrm{M}$ within 2$10 \mathrm{~mm}$ long segment of a capillary with a diameter comparable to RBC size $(7 \mu \mathrm{m})$. Thus, a miniscule fraction of the circulating pre-senescent cells, e.g., those prior to their processing in the splenic system could constitute a sufficient pool of RBCs available for intravascular hemolytic ATP release and blood flow control. Contribution of the oldest RBCs showing so called terminal density reversal and the role of the nonselective cationic channels in the sustained elevation of $\mathrm{Ca}^{2+}$ and triggering of hemolysis 
should be also considered (Lew and Tiffert, 2013; Thomas, 2014).

\section{Search for Upstream $\mathrm{PO}_{2}$ Sensors and Downstream Intermediates of $\mathrm{PO}_{2}$-Dependent Signaling}

Hemoglobin is the only known $\mathrm{O}_{2}$-binding protein in erythrocytes. Keeping this in mind, the reversible association of oxygenated and/or deoxygenated hemoglobin (oxyHb and deoxyHb, respectively) with downstream intermediates of intracellular signaling might be considered as a mechanism of triggering $\mathrm{PO}_{2}$-dependent erythrocyte responses. Indeed, in cell-free experiments it was shown that hemoglobin binds to the cytoplasmic domain of band 3 (cdb3) (Cassoly, 1983; Low et al., 1984) also known as anion exchanger (AE1, SLC4A1), i.e., the major integral protein of erythrocytes membrane, playing a key role in anion transport and the organization of membrane cytoskeleton (Reithmeier et al., 2016). Importantly, both in human and mice the affinity of $\mathrm{cdb} 3$ for deoxyHb is much higher than for oxyHb (Walder et al., 1984; Sega et al., 2012, 2015).

Downstream intermediates of $\mathrm{PO}_{2}$-dependent erythrocyte responses remain poorly understood (Figure 2). Stefanovic and co-workers has demonstrated that oxygentation strengthens band 3-ankyrin interactions, thereby stabilizing the erythrocyte membrane during turbulent flow from the lungs to the capillary beds (Stefanovic et al., 2013). Deoxygenation displaces ankyrin from band 3 releasing the spectrin/actin cytoskeleton from the membrane. This weakening of membranecytoskeleton interactions could increase RBC deformability enabling deoxygenated RBCs move more efficiently through narrow capillaries. Prolonged deoxygenation, on the other hand, could increase membrane propensity to rupture due to diminished mechanical support by the cytoskeleton. Indeed, theoretical considerations of membrane stability and pore formation in a lipid bilayer showed importance of cytoskeletal network in stabilizing the membrane against pore growth by reducing the surface tension (Sung and Park, 1997).

It is important to note that when oxygenated RBCs enter a region of low $\mathrm{PO}_{2}$ the full-scale saturation of hemoglobin deoxygenation occurs within $25 \mathrm{~ms}$ (Ellsworth et al., 2016). Therefore, we constructed a special chamber allowing isolation of RBC ghosts in control and deoxygenated conditions and found a $\sim 2$-fold elevation of $\sim 60 \mathrm{kDa}$ membranebound protein content under deoxygenated conditions (Luneva et al., 2016). Currently, we employ proteomics technology for identification of full set of proteins whose interaction with RBC membrane is affected by hypoxia. This approach should lead to

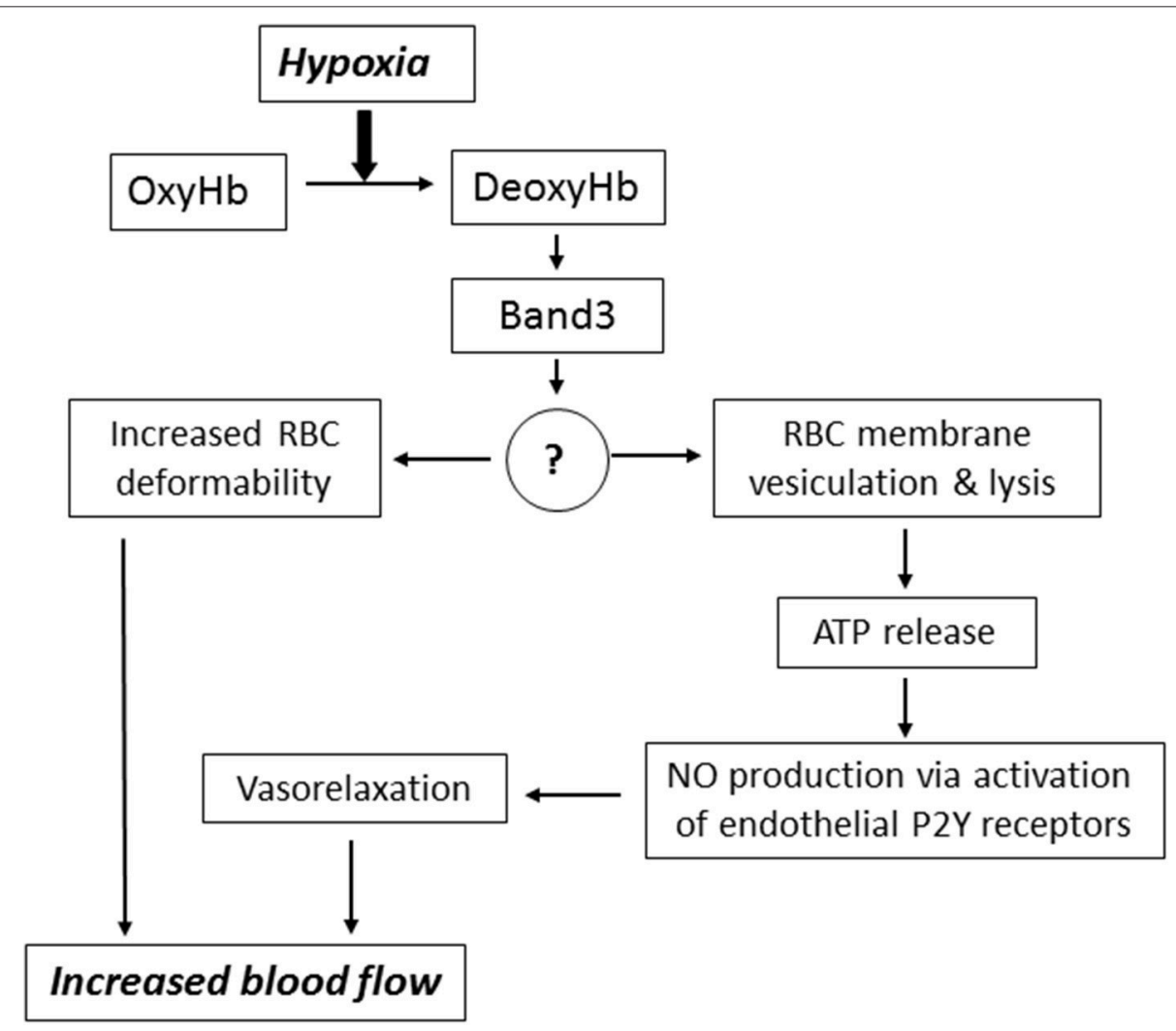

FIGURE 2 | Mechanisms underlying the implication of RBC in blood flow regulation. Hypoxia leads to accumulation of deoxyhemoglobin (DeoxyHb) and its interaction with cytoplasmic domain of anion exchanger (Band 3 protein). This interaction triggers signaling via changes in the content of unknown membrane-bound proteins (?) resulting in increased RBC deformability, membrane vesiculation and ATP release via hemolysis. For more details, see text. 
identification of downstream intermediates involved in hypoxiainduced ATP release mediated by diminished RBC membrane integrity.

\section{FUTURE RESEARCH DIRECTIONS}

Several issues remain unsettled and require further investigations. Among them the question remains if besides hemolysis as the primary release mechanisms, are there conditions or stimuli that would induce regulated non-lytic ATP release from RBC and what pathway it could involve? What might be the contribution of young immature erythrocytes (reticulocytes) to such release? With their residual intracellular structures could they release ATP via exocytosis? While modest in relative terms $(0.5-2.5 \%)$ these cells constitute a significant cellular pool $\left(\sim 4.5 \times 10^{4}\right.$ cells $\left./ \mu \mathrm{l}\right)$ with plenty of ATP for local purinergic signaling. If conductive release pathway would

\section{REFERENCES}

Akopova, I., Tatur, S., Grygorczyk, M., Luchowski, R., Gryczynski, I., Gryczynski, Z., et al. (2012). Imaging exocytosis of ATP-containing vesicles with TIRF microscopy in lung epithelial A549 cells. Purinergic Signal. 8, 59-70. doi: 10.1007/s11302-011-9259-2

Alaarg, A., Schiffelers, R. M., van Solinge, W. W., and van Wijk, R. (2013). Red blood cell vesiculation in hereditary hemolytic anemia. Front. Physiol. 4:365. doi: 10.3389/fphys.2013.00365

Albuisson, J., Murthy, S. E., Bandell, M., Coste, B., Louis-Dit-Picard, H., Mathur, J., et al. (2013). Dehydrated hereditary stomatocytosis linked to gain-of-function mutations in mechanically activated PIEZO1 ion channels. Nat. Commun. 4:1884. doi: $10.1038 /$ ncomms 2899

Báthori, G., Parolini, I., Szabó, I., Tombola, F., Messina, A., Oliva, M., et al. (2000). Extramitochondrial porin: facts and hypotheses. J. Bioenerg. Biomembr. 32, 79-89. doi: 10.1023/A:1005516513313

Bergfeld, G. R., and Forrester, T. (1992). Release of ATP from human erythrocytes in response to a brief period of hypoxia and hypercapnia. Cardiovasc. Res. 26, 40-47. doi: $10.1093 / \mathrm{cvr} / 26.1 .40$

Bogdanova, A., Berenbrink, M., and Nikinmaa, M. (2009). Oxygendependent ion transport in erythrocytes. Acta Physiol. 195, 305-319. doi: 10.1111/j.1748-1716.2008.01934.x

Brugnara, C., Gee, B., Armsby, C. C., Kurth, S., Sakamoto, M., Rifai, N., et al. (1996). Therapy with oral clotrimazole induces inhibition of the Gardos channel and reduction of erythrocytes dehydration in patients with sicle cell disease. J. Clin. Invest. 97, 1227-1234. doi: 10.1172/JCI118537

Cassoly, R. (1983). Quantitative analysis of the association of human hemoglobin with the cytoplasmic fragment of band 3 protein. J. Biol. Chem. 258, 3859-3864.

Chiu, Y. H., Ravichandran, K. S., and Bayliss, D. A. (2014). Intrinsic properties and regulation of Pannexin 1 channel. Channels 8, 103-109. doi: $10.4161 /$ chan. 27545

Chu, H., McKenna, M. M., Krump, N. A., Zheng, S., Mendelsohn, L., Thein, S. L., et al. (2016). Reversible binding of hemoglobin to band 3 constitutes the molecular switch that mediates $\mathrm{O} 2$ regulation of erythrocyte properties. Blood 128, 2708-2716. doi: 10.1182/blood-2016-01-692079

Ciana, A., Achilli, C., Gaur, A., and Minetti, G. (2017). Membrane Remodelling and vesicle formation during ageing of human red blood cells. Cell. Physiol. Biochem. 42, 1127-1138. doi: 10.1159/000478768

Cinar, E., Zhou, S., DeCourcey, J., Wang, Y., Waugh, R. E., and Wan, J. (2015). Piezol regulates mechanotransductive release of ATP from human RBCs. Proc. Natl. Acad. Sci. U.S.A. 112, 11783-11788. doi: 10.1073/pnas.1507 309112

Colombini, M. (2012). VDAC structure, selectivity, and dynamics. Biochim. Biophys. Acta 1818, 1457-1465. doi: 10.1016/j.bbamem.2011.12.026 be involved what could be the mechanism allowing selective permeation of ATP but preventing massive influx of $\mathrm{Ca}^{2+}$ and $\mathrm{Na}^{+}$? Finally, much remains to be learned about the downstream intermediates involved in hypoxia-induced membrane fragility, membrane microdefects, lytic pore formation and associated ATP release.

\section{AUTHOR CONTRIBUTIONS}

All authors listed have made a substantial, direct and intellectual contribution to the work, and approved it for publication.

\section{ACKNOWLEDGMENTS}

This work was supported by grants from the Canadian Institutes of Health Research (MOP64364 to RG) and the Russian Scientific Foundation (RNF \#16-15-10026 to SO).
Dando, R., and Roper, S. D. (2009). Cell-to-cell communication in intact taste buds through ATP signalling from pannexin 1 gap junction hemichannels. J. Physiol. 587(Pt 24), 5899-5906. doi: 10.1113/jphysiol.2009.180083

D'hondt, C., Ponsaerts, R., De Smedt, H., Vinken, M., De Vuyst, E., De Bock, M., et al. (2010). Pannexin channels in ATP release and beyond: An unexpected rendezvous at the endoplasmic reticulum. Cell Signal 23, 305-316. doi: 10.1016/j.cellsig.2010.07.018

Dietrich, H. H., Ellsworth, M. L., Sprague, R. S., and Dacey, R. G. Jr. (2000). Red blood cell regulation of microvascular tone through adenosine triphosphate. Am. J. Physiol. Heart Circ. Physiol. 278, H1294-H1298. doi: 10.1152/ajpheart.2000.278.4.H1294

Diez-Silva, M., Dao, M., Han, J., Lim, C. T., and Suresh, S. (2010). Shape and biomechanical characteristics of human red blood cells in health and disease. MRS Bull. 35, 382-388. doi: 10.1557/mrs2010.571

Durocher, J. R., Payne, R. C., and Conrad, M. E. (1975). Role of sialic acid in erythrocyte survival. Blood 45, 11-20.

Ellsworth, M. L., Ellis, C. G., Goldman, D., Stephenson, A. H., Dietrich, H. H., and Sprague, R. S. (2009). Erythrocytes: oxygen sensors and modulators of vascular tone. Physiology 24, 107-116. doi: 10.1152/physiol.00038.2008

Ellsworth, M. L., Ellis, C. G., and Sprague, R. S. (2016). Role of erythrocyte-released ATP in the regulation of microvascular oxygen supply in skeletal muscle. Acta Physiol. 216, 265-276. doi: 10.1111/apha.12596

Ellsworth, M. L., Forrester, T., Ellis, C. G., and Dietrich, H. H. (1995). The erythrocyte as a regulator of vascular tone. Am. J. Physiol. 269(6 Pt 2), H2155-H2161.

Ellsworth, M. L., and Sprague, R. S. (2012). Regulation of blood flow distribution in skeletal muscle: role of erythrocyte-released ATP. J. Physiol. 590(Pt 20), 4985-4991. doi: 10.1113/jphysiol.2012.233106

Fischer, D. J., Torrence, N. J., Sprung, R. J., and Spence, D. M. (2003). Determination of erythrocyte deformability and its correlation to cellular ATP release using microbore tubing with diameters that approximate resistance vessels in vivo. Analyst 128, 1163-1168. doi: 10.1039/b308225n

Forrester, T. (1972). An estimate of adenosine triphosphate release into the venous effluent from exercising human forearm muscle. J. Physiol. 224, 611-628. doi: 10.1113/jphysiol.1972.sp009915

Forsyth, A. M., Braunmüller, S., Wan, J., Franke, T., and Stone, H. A. (2012). The effects of membrane cholesterol and simvastatin on red blood cell deformability and ATP release. Microvasc. Res. 83, 347-351. doi: 10.1016/j.mvr.2012.02.004

Forsyth, A. M., Wan, J., Owrutsky, P. D., Abkarian, M., and Stone, H. A. (2011). Multiscale approach to link red blood cell dynamics, shear viscosity, and ATP release. Proc. Natl. Acad. Sci. U.S.A. 108, 10986-10991. doi: 10.1073/pnas.1101315108

González-Alonso, J., Olsen, D. B., and Saltin, B. (2002). Erythrocyte and the regulation of human skeletal muscle blood flow and 
oxygen delivery: role of circulating ATP. Circ. Res. 91, 1046-1055. doi: 10.1161/01.RES.0000044939.73286.E2

Greenwalt, T. J. (2006). The how and why of exocytic vesicles. Transfusion 46, 143-152. doi: 10.1111/j.1537-2995.2006.00692.x

Grygorczyk, R., and Hanrahan, J. W. (1997a). CFTR-independent ATP release from epithelial cells triggered by mechanical stimuli. Am. J. Physiol. 272(3 Pt 1), C1058-C1066.

Grygorczyk, R., and Hanrahan, J. W. (1997b). Cystic fibrosis transmembrane conductance regulator and adenosine triphosphate [response]. Science 275, $1325-1326$.

Grygorczyk, R., Tabcharani, J. A., and Hanrahan, J. W. (1996). CFTR channels expressed in CHO cells do not have detectable ATP conductance. J. Membr. Biol. 151, 139-148. doi: 10.1007/s002329900065

Hazama, A., Shimizu, T., Ando-Akatsuka, Y., Hayashi, S., Tanaka, S., Maeno, E., et al. (1999). Swelling-induced, CFTR-independent ATP release from a human epithelial cell line: lack of correlation with volume-sensitive $\mathrm{Cl}$ - channels. J. Gen. Physiol. 114, 525-533. doi: 10.1085/jgp.114.4.525

Hoffman, J. F., Dodson, A., Wickrema, A., and Dib-Hajj, S. D. (2004). Tetrodotoxin-sensitive $\mathrm{Na}^{+}$channels and muscarinic and purinergic receptors identified in human erythroid progenitor cells and red blood cell ghosts. Proc. Natl. Acad. Sci. U.S.A. 101, 12370-12374. doi: 10.1073/pnas.0404228101

Huang, Y. X., Wu, Z. J., Mehrishi, J., Huang, B. T., Chen, X. Y., Zheng, X. J., et al. (2011). Human red blood cell aging: correlative changes in surface charge and cell properties. J. Cell. Mol. Med. 15, 2634-2642. doi: 10.1111/j.1582-4934.2011.01310.x

Jensen, F. B. (2009). The dual roles of red blood cells in tissue oxygen delivery: oxygen carriers and regulator of local blood flow. J. Exp. Biol. 212, 3387-3393. doi: $10.1242 /$ jeb. 023697

Keller, A. S., Diederich, L., Panknin, C., DeLalio, L. J., Drake, J. C., Sherman, R., et al. (2017). Possible roles for ATP release from RBCs exclude the cAMP-mediated Panx1 pathway. Am. J. Physiol. Cell Physiol. 313, C593-C603. doi: 10.1152/ajpcell.00178.2017

Kirby, B. S., Crecelius, A. R., Voyles, W. F., and Dinenno, F. A. (2012). Impaired skeletal muscle blood flow control with advancing age in humans: attenuated ATP release and local vasodilation during erythrocyte deoxygenation. Circ. Res. 111, 220-230. doi: 10.1161/CIRCRESAHA.112.269571

Kirby, B. S., Hanna, G., Hendargo, H. C., and McMahon, T. J. (2014). Restoration of intracellular ATP production in banked red blood cells improves inducible ATP export and suppresses RBC-endothelial adhesion. Am. J. Physiol. Heart Circ. Physiol. 307, H1737-H1744. doi: 10.1152/ajpheart.00542.2014

Kirby, B. S., Schwarzbaum, P. J., Lazarowski, E. R., Dinenno, F. A., and McMahon, T. J. (2015). Liberation of ATP secondary to hemolysis is not mutually exclusive of regulated export. Blood 125, 1844-1845. doi: 10.1182/blood-2014-11-609610

Lazarowski, E. R., Sesma, J. I., Seminario-Vidal, L., and Kreda, S. M. (2011). Molecular mechanisms of purine and pyrimidine nucleotide release. $A d v$. Pharmacol. 61, 221-261. doi: 10.1016/B978-0-12-385526-8.00008-4

Lew, V. L., and Tiffert, T. (2013). The terminal density reversal phenomenon of aging human red blood cells. Front. Physiol. 4:171. doi: 10.3389/fphys.2013.00171

Li, C., Ramjeesingh, M., and Bear, C. E. (1996). Purified cystic fibrosis transmembrane conductance regulator (CFTR) does not function as an ATP channel. J. Biol. Chem. 271, 11623-11626. doi: 10.1074/jbc.271.20.11623

Li, F., Chan, C. U., and Ohl, C. D. (2013). Yield strength of human erythrocyte membranes to impulsive stretching. Biophys. J. 105, 872-879. doi: 10.1016/j.bpj.2013.06.045

Low, P. S., Westfall, M. A., Allen, D. P., and Appel, K. C. (1984). Characterization of the reversible conformational equilibrium of the cytoplasmic domain of erythrocyte membrane band 3. J. Biol. Chem. 259, 13070-13076.

Luneva, O. G., Sidorenko, S. V., Maksimov, G. V., Grygorczyk, R., and Orlov, S. N. (2015). Erythrocytes as regulators of blood vessel tone. Biochem. Suppl. Ser. A Membr. Cell Biol. 9, 161-171. doi: 10.1134/S1990747815040078

Luneva, O. G., Sidorenko, S. V., Ponomarchuk, O. O., Tverskoy, A. M., Cherkashin, A. A., Rodnenkov, O. V., et al. (2016). Deoxygenation affects composition of membrane-bound proteins in human erythrocytes. Cell. Physiol. Biochem. 39, 81-88. doi: 10.1159/000445607

Ma, W., Compan, V., Zheng, W., Martin, E., North, R. A., Verkhratsky, A., et al. (2012). Pannexin 1 forms an anion-selective channel. Pflugers Arch. 463, 585-592. doi: 10.1007/s00424-012-1077-z
Ma, W., Hui, H., Pelegrin, P., and Surprenant, A. (2009). Pharmacological characterization of pannexin-1 currents expressed in mammalian cells. $J$. Pharmacol. Exp. Ther. 328, 409-418. doi: 10.1124/jpet.108.146365

Mairbäurl, H. (2013). Red blood cells in sports: effects of exercise and training on oxygen supply by red blood cells. Front. Physiol. 4:332. doi: 10.3389 /fphys.2013.00332

Mairbäurl, H., Ruppe, F. A., and Bärtsch, P. (2013). Role of hemolysis in red cell adenosine triphosphate release in simulated exercise conditions in vitro. Med. Sci. Sports Exerc. 45, 1941-1947. doi: 10.1249/MSS.0b013e318296193a

Mao, T. Y., Fu, L. L., and Wang, J. S. (2011). Hypoxic exercise training causes erythrocyte senescence and rheological dysfunction by depressed Gardos channel activity. J. Appl. Physiol. (1985) 111, 382-391. doi: 10.1152/japplphysiol.00096.2011

Mehdi, M. M., Singh, P., and Rizvi, S. I. (2012). Erythrocyte sialic acid content during aging in humans: correlation with markers of oxidative stress. Dis. Markers 32, 179-186. doi: 10.1155/2012/293429

Messana, I., Orlando, M., Cassiano, M., Pennacchietti, L., Zuppi, C., Castagnola, M., et al. (1996). Human erythrocyte metabolism is modulated by the $\mathrm{O}_{2}$-linked transition of hemoglobin. FEBS Lett. 390, 25-28. doi: 10.1016/0014-5793(96)00624-2

Mohandas, N., and Gallagher, P. G. (2008). Red cell membrane: past, present, and future. Blood 112, 3939-3948. doi: 10.1182/blood-2008-07-161166

Montalbetti, N., Leal Denis, M. F., Pignataro, O. P., Kobatake, E., Lazarowski, E. R., and Schwarzbaum, P. J. (2011). Homeostasis of extracellular ATP in human erythrocytes. J. Biol. Chem. 286, 38397-38407. doi: 10.1074/jbc.M111. 221713

Nikinmaa, M. (2002). Oxygen-dependent cellular functions - why fishes and aquatic envirinment are a prime choice of study. Comp. Biochem. Physiol. A Mol. Integr. Physiol. 2133, 1-16. doi: 10.1016/S1095-6433(02)00132-0

Olearczyk, J. J., Stephenson, A. H., Lonigro, A. J., and Sprague, R. S. (2001). Receptor-mediated activation of the heterotrimeric G-protein Gs results in ATP release from erythrocytes. Med. Sci. Monit. 7, 669-674.

Orbach, A., Zelig, O., Yedgar, S., and Barshtein, G. (2017). Biophysical and biochemical markers of red blood cell fragility. Transfus. Med. Hemother. 44, 183-187. doi: 10.1159/000452106

Park, Y., Best, C. A., Auth, T., Gov, N. S., Safran, S. A., Popescu, G., et al. (2010). Metabolic remodeling of the human red blood cell membrane. Proc. Natl. Acad. Sci. U.S.A. 107, 1289-1294. doi: 10.1073/pnas.0910785107

Praetorius, H. A., and Leipziger, J. (2009). ATP release from non-excitable cells. Purinergic Signal. 5, 433-446. doi: 10.1007/s11302-009-9146-2

Price, A. K., Fischer, D. J., Martin, R. S., and Spence, D. M. (2004). Deformationinduced release of ATP from erythrocytes in a poly(dimethylsiloxane)-based microchip with channels that mimic resistance vessels. Anal. Chem. 76, 4849-4855. doi: 10.1021/ac0495992

Qiu, F., Wang, J., Spray, D. C., Scemes, E., and Dahl, G. (2011). Two non-vesicular ATP release pathways in the mouse erythrocyte membrane. FEBS Lett. 585, 3430-3435. doi: 10.1016/j.febslet.2011.09.033

Ransford, G. A., Fregien, N., Qiu, F., Dahl, G., Conner, G. E., and Salathe, M. (2009). Pannexin 1 Contributes to ATP Release in airway epithelia. Am. J. Respir. Cell Mol. Biol. 41, 525-534. doi: 10.1165/rcmb.20080367OC

Reddy, M. M., Quinton, P. M., Haws, C., Wine, J. J., Grygorczyk, R., Tabcharani, J. A., et al. (1996). Failure of the cystic fibrosis transmembrane conductance regulator to conduct ATP. Science 271, 1876-1879.

Reisin, I. L., Prat, A. G., Abraham, E. H., Amara, J. F., Gregory, R. J., Ausiello, D. A., et al. (1994). The cystic fibrosis transmembrane conductance regulator is a dual ATP and chloride channel. J. Biol. Chem. 269, 20584-20591.

Reithmeier, R. A., Casey, J. R., Kalli, A. C., Sansom, M. S. P., Alguel, Y., and Iwata, S. (2016). Band 3, the human red cell chloride/bicarbonate exchanger (AE1, SLC4A1), in a structural context. Biochim. Biophys. Acta 1858, 1507-1532. doi: 10.1016/j.bbamem.2016.03.030

Romanello, M., Pani, B., Bicego, M., and D’Andrea, P. (2001). Mechanically induced ATP release from human osteoblastic cells. Biochem. Biophys. Res. Commun. 289, 1275-1281. doi: 10.1006/bbrc.2001.6124

Romanov, R. A., Bystrova, M. F., Rogachevskaya, O. A., Sadovnikov, V. B., Shestopalov, V. I., and Kolesnikov, S. S. (2012). The ATP permeability of pannexin 1 channels in a heterologous system and in mammalian taste cells is dispensable. J. Cell Sci. 125(Pt 22), 5514-5523. doi: 10.1242/jcs.111062 
Rostovtseva, T., and Colombini, M. (1997). VDAC channels mediate and gate the flow of ATP: implications for the regulation of mitochondrial function. Biophys. J. 72, 1954-1962. doi: 10.1016/S0006-3495(97)78841-6

Sabirov, R. Z., Merzlyak, P. G., Okada, T., Islam, M. R., Uramoto, H., Mori, T., et al. (2017). The organic anion transporter SLCO2A1 constitutes the core component of the Maxi-Cl channel. EMBO J. 36, 3309-3324. doi: $10.15252 / \mathrm{embj} .201796685$

Sabirov, R. Z., and Okada, Y. (2005). ATP release via anion channels. Purinergic Signal. 1, 311-328. doi: 10.1007/s11302-005-1557-0

Sabirov, R. Z., Sheiko, T., Liu, H., Deng, D., Okada, Y., and Craigen, W. J. (2006). Genetic demonstration that the plasma membrane maxianion channel and voltage-dependent anion channels are unrelated proteins. J. Biol. Chem. 281, 1897-1904. doi: 10.1074/jbc.M509482200

Sega, M. F., Chu, H., Christian, J. A., and Low, P. S. (2015). Fluorescence assay of the interaction between hemoglobin and the cytoplasmic domain of erythrocyte membrane band 3. Blood Cells Mol. Dis. 55, 266-271. doi: 10.1016/j.bcmd.2015.07.004

Sega, M. F., Chu, H., Christian, J., and Low, P. S. (2012). Interaction of deoxyhemoglobin with the cytoplasmic domain of murine erythrocyte band 3 . Biochemistry 51, 3264-3272. doi: 10.1021/bi201623v

Shaskey, D. J., and Green, G. A. (2000). Sports haematology. Sports Med. 29, 27-38. doi: 10.2165/00007256-200029010-00003

Sikora, J., Orlov, S. N., Furuya, K., and Grygorczyk, R. (2014). Hemolysis is a primary ATP-release mechanism in human erythrocytes. Blood 124, 2150-2157. doi: 10.1182/blood-2014-05-572024

Sikora, J., Orlov, S. N., Furuya, K., and Grygorczyk, R. (2015). Response: hemolysis is a primary and physiologically relevant ATP release mechanism in human erythrocytes. Blood 125, 1845-1846. doi: 10.1182/blood-2015-01-622159

Sprague, R. S., Ellsworth, M. L., Stephenson, A. H., Kleinhenz, M. E., and Lonigro, A. J. (1998). Deformation-induced ATP release from red blood cells requires CFTR activity. Am. J. Physiol. 275(5 Pt 2), H1726-H1732.

Sprague, R. S., Ellsworth, M. L., Stephenson, A. H., and Lonigro, A. J. (1996). ATP: the red blood cell link to $\mathrm{NO}$ and local control of the pulmonary circulation. Am. J. Physiol. 271(6 Pt 2), H2717-H2722.

Sprague, R. S., Stephenson, A. H., and Ellsworth, M. L. (2007). Red not dead: signaling in and from erythrocytes. Trends Endocrinol. Metab. 18, 350-355. doi: $10.1016 /$ j.tem.2007.08.008

Sridharan, M., Adderley, S. P., Bowles, E. A., Egan, T. M., Stephenson, A. H., Ellsworth, M. L., et al. (2010). Pannexin 1 is the conduit for low oxygen tensioninduced ATP release from human erythrocytes. Am. J. Physiol. Heart Circ. Physiol. 299, H1146-H1152. doi: 10.1152/ajpheart.00301.2010

Sridharan, M., Bowles, E. A., Richards, J. P., Krantic, M., Davis, K. L., Dietrich, K. A., et al. (2012). Prostacyclin receptor-mediated ATP release from erythrocytes requires the voltage-dependent anion channel. Am. J. Physiol. Heart Circ. Physiol. 302, H553-H559. doi: 10.1152/ajpheart.00998.2011

Stefanovic, M., Puchulu-Campanella, E., Kodippili, G., and Low, P. S. (2013). Oxygen regulates the band 3-ankyrin bridge in the human erythrocyte membrane. Biochem. J. 449, 143-150. doi: 10.1042/BJ20120869

Sugita, M., Yue, Y., and Foskett, J. K. (1998). CFTR Cl- channel and CFTRassociated ATP channel: distinct pores regulated by common gates. EMBO J. 17, 898-908. doi: $10.1093 /$ emboj/17.4.898
Sung, W., and Park, P. J. (1997). Dynamics of pore growth in membranes and membrane stability. Biophys. J. 73, 1797-1804. doi: 10.1016/S0006-3495(97)78210-9

Thomas, S. L. (2014). Intravascular hemolysis: the sacrifice of few. Blood 124, 2011-2012. doi: 10.1182/blood-2014-08-595447

Tiffert, T., Etzion, Z., Bookchin, R. M., and Lew, V. L. (1993). Effects of deoxygenation on active and passive $\mathrm{Ca}^{2+}$ transport and cytoplasmic $\mathrm{Ca}^{2+}$ buffering in normal human red cells. J. Physiol. 464, 529-544. doi: 10.1113/jphysiol.1993.sp019649

Viallat, A., and Abkarian, M. (2014). Red blood cell: from its mechanics to its motion in shear flow. Int. J. Lab. Hematol. 36, 237-243. doi: 10.1111/ijlh. 12233

Walder, J. A., Chatterjee, R., Steck, T. L., Low, P. S., Musso, G. F., Kaiser, E. T., et al. (1984). The interaction of hemoglobin with the cytoplasmic domain of band 3 of the human eyrthrocyte membrabe. J. Biol. Chem. 259, 10238-10246.

Wan, J., Forsyth, A. M., and Stone, H. A. (2011). Red blood cell dynamics: from cell deformation to ATP release. Integr. Biol. 3, 972-981. doi: 10.1039/clib $00044 \mathrm{f}$

Wang, L., Olivecrona, G., Götberg, M., Olsson, M. L., Winzell, M. S., and Erlinge, D. (2005). ADP acting on P2Y13 receptors is a negative feedback pathway for ATP release from human red blood cells. Circ. Res. 96, 189-196. doi: 10.1161/01.RES.0000153670.07559.E4

Watt, W. C., Lazarowski, E. R., and Boucher, R. C. (1998). Cystic fibrosis transmembrane regulator-independent release of ATP. Its implications for the regulation of $\mathrm{P} 2 \mathrm{Y} 2$ receptors in airway epithelia. J. Biol. Chem. 273, 14053-14058. doi: 10.1074/jbc.273.22.14053

Wei, H. S., Kang, H., Rasheed, I. Y., Zhou, S., Lou, N., Gershteyn, A., et al. (2016). Erythrocytes are oxygen-sensing regulators of the cerebral microcirculation. Neuron 91, 851-862. doi: 10.1016/j.neuron.2016.07.016

Willekens, F. L., Werre, J. M., Groenen-Döpp, Y. A., RoerdinkholderStoelwinder, B., de Pauw, B., and Bosman, G. J. (2008). Erythrocyte vesiculation: a self-protective mechanism? Br. J. Haematol. 141, 549-556. doi: 10.1111/j.1365-2141.2008.07055.x

Yedgar, S., Koshkaryev, A., and Barshtein, G. (2002). The red blood cell in vascular occlusion. Pathophysiol. Haemost. Thromb. 32, 263-268. doi: $10.1159 / 000073578$

Zarychanski, R., Schulz, V. P., Houston, B. L., Maksimova, Y., Houston, D. S., Smith, B., et al. (2012). Mutations in the mechanotransduction protein PIEZO1 are associated with hereditary xerocytosis. Blood 120, 1908-1915. doi: 10.1182/blood-2012-04-422253

Conflict of Interest Statement: The authors declare that the research was conducted in the absence of any commercial or financial relationships that could be construed as a potential conflict of interest.

Copyright (c) 2017 Grygorczyk and Orlov. This is an open-access article distributed under the terms of the Creative Commons Attribution License (CC BY). The use, distribution or reproduction in other forums is permitted, provided the original author(s) or licensor are credited and that the original publication in this journal is cited, in accordance with accepted academic practice. No use, distribution or reproduction is permitted which does not comply with these terms. 